\title{
Shape Characterization of Extracted and Simulated Tumor Samples using Topological and Geometric Measures
}

\author{
Markus Rohrschneider*, Gerik Scheuermann*, Stefan Hoehme ${ }^{\dagger}$, Dirk Drasdo
}

\begin{abstract}
The prognosis of cancer patients suffering from solid tumors significantly depends on the developmental stage of the tumor. For cervix carcinoma the prognosis is better for compact shapes than for diffusive shapes since the latter may already indicate invasion, the stage in tumor progression that precedes the formation of metastases. In this paper, we present methods for describing and evaluating tumor objects and their surfaces based on topological and geometric properties. For geometry, statistics of the binary object's distance transform are used to evaluate the tumor's invasion front. In addition, a simple compactness measure is adapted to $3 \mathrm{D}$ images and presented to compare different types of tumor samples. As a topological measure, the Betti numbers are calculated of voxelized tumor objects based on a medial axis transform. We further illustrate how these geometric and topological properties can be used for a quantitative comparison of histological material and singlecell-based tumor growth simulations.
\end{abstract}

\section{INTRODUCTION}

In industrialized nations, malignant tumors are the secondmost frequent cause of death after cardiovascular diseases. For the development of successful therapy strategies, a thorough understanding of the biological mechanisms of tumor growth is essential. Invasive cancer is the result of many mutations. While in its direct precursor state, a tumor insitu is well separated from the surrounding tissue, cells of an invasive cancer invade the surrounding tissue and secrete angiogenesis factors that trigger the formation of new blood vessels sprouting towards the tumor. There is some evidence for correlation between the tumor's morphology and its malignity (e.g. [22], [4]), and for the proper choice of therapy maximum knowledge about the tumor state is required.

Besides animal models and clinical studies, mathematical models are increasingly used to analyze the possible mechanisms leading to growth and invasion, and suggest possible therapy strategies. The aim of this work is to establish measures to distinguish tumor shapes and quantify shape differences as a possible basis of clinical assessments and for comparisons of tumor growth simulations with histological data. We demonstrate our measures with tissue samples and simulated (model) tumors. The effects of the model parameters on the resulting morphology were examined and characterized using different shape descriptors of geometry and topology. The applied geometric methods include

* M. Rohrschneider and G. Scheuermann are with the Image and Signal Processing Group at the University of Leipzig, Germany \{rohrschneider|scheuermann\}@informatik.uni-leipzig.de

$\dagger \mathrm{S}$. Hoehme is with the Interdisciplinary Centre of Bioinformatics (IZBI), University of Leipzig, Germany

† D. Drasdo is with the Institut National de Recherche en Informatique et en Automatique (INRIA), Unit Rocquencourt, France and IZBI, Leipzig statistics on distance values between the medial axis and the object boundary, and compactness considerations to define morphological complexity. Additionally, we developed topological methods to compute the Betti numbers on 3D discretized binary objects.

\section{RELATED WORK}

Shape description is one of the major disciplines of image processing. Topological shape descriptors, such as the Betti numbers, are commonly used to describe isosurfaces of $3 \mathrm{D}$ scalar fields [16].

Skeletons can be used for a large variety of tasks including visualization improvement, animations, virtual navigation, mesh generation etc. [7]. Skeletons can be computed via continuous or discrete approaches [2]. In the continuous case, the skeleton is approximated using the Voronoi graph of a discrete sample set of the object boundary. Amenta et al. [1] compute the medial axis from noisy point clouds to reconstruct an object's surface. The medial axis has been used to represent shape in various works [2], [18], [13]. Kruszynski et al. use the medial axis to describe and quantify coral structures. Many methods exist to compute the skeleton in discrete environments, e.g. by morphological thinning algorithms [15], [3], and by methods based on the distance transform [19].

\section{TUMOR GROWTH SIMULATIONS}

For the simulated tumors we use a model in which each cell is represented explicitly and parameterized by cellbiological, cell-biophysical and cell-kinetic quantities [8]. In this approach, an isolated cell is modeled as elastic homogeneous isotropic sphere of radius $R$, which slightly flattens at the contact region if attached to another cell and deforms into a dumb-bell with radius $R$ and axis length $a$ during cell growth in small successive growth steps $\delta a \ll$ $R$. Cells can migrate and adhere, and consume nutrients from the environment. The forces between adjacent cells are modeled by the Johnson-Kendall-Roberts model for isotropic homogeneous strongly adhesive spheres [12] that shows a characteristic hysteresis behavior between the attachment and detachment process [9]. The interaction force contains information about the Young modulus $E$, the Poisson number $v$, and the density of adhesion molecules $\rho$. Cells are able to grow and divide above a threshold nutrient concentration if they are not compressed or deformed below a certain threshold size. If the local nutrient concentration falls below a defined threshold, cells die (necrosis) and are removed from the simulations since in this case they fragmentize into small 
pieces. We model the migration of cells by the Metropolis algorithm and a proper definition of time scales. A cell in isolation performs a random-walk-like movement while in the neighborhood of other cells it tends to move into the direction which minimizes the free energy. We quantify the migration activity of a cell by its diffusion constant $D$ in isolation. We perform a number of successive migration and orientation trials between two successive growth trials. The trials are accepted with probability $\min \left(1, \exp \left\{-\Delta V / F_{T}\right\}\right.$. $F_{T}$ is a parameter that controls the cell activity: it may be compared to the thermal energy $k_{B} T$ in fluids $\left(k_{B}\right.$ : Boltzman constant, $T$ : temperature). Together with the choice of step sizes for growth, orientation change, and migration, our algorithm mimics a multi-cellular configuration changing with time. The step sizes are chosen in such a way, that the simulation reflects a realistic growth scenario. (The details of our model are explained in [8])

We recently used this single-cell based model to study tumor spheroid growth in liquid suspension [8], which has been extensively studied experimentally [21], [14] (for an overview of tumor growth models, please see Ref. [8] and references therein). Here, we study growing tumors in a tissue-like medium composed of cells to analyze the influence of an embedding medium on the tumor morphology (for a simulation example, see Fig. 1)

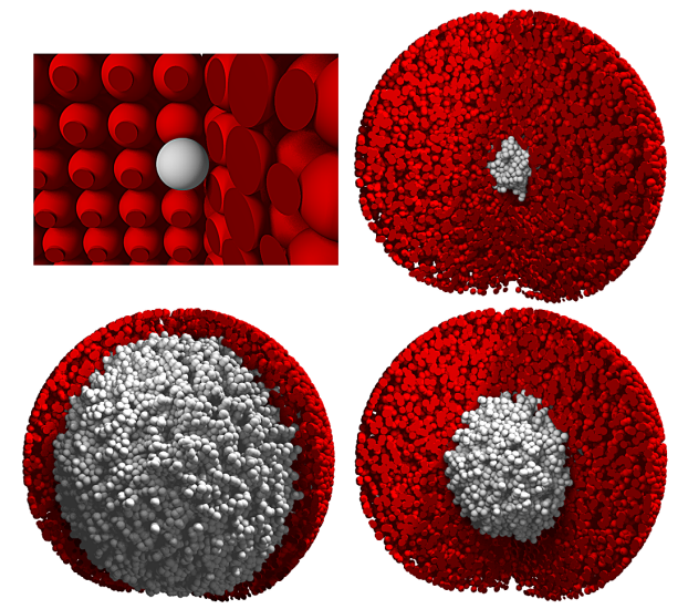

Fig. 1. Typical simulated tumor growth scenario. Red: embedding cells, white: cells of the expanding clone. The embedding cells are initially placed on the nodes of a square lattice and subsequently relaxed before the growth of the embedded clone is started.

The embedding medium was modeled as non-dividing cells with the same parameters as the dividing cell clone with the following exceptions: (1) "motX" within the name of the dataset denotes that $D \rightarrow D / X$ with $D$ being the Diffusion constant mentioned above, (2) except of the dataset "id100_mot1_adh" the embedding cells do not adhere. The id-value refers to the initial distance of embedding cells which is $l$ for id100 and $l=1.2(=120 / 100)$ for $i d 120$. For selected parameter sets, we have validated that the results do not change if we replace the embedding cells by granular particles with the same physical properties, but with only passive movement (i.e., no capability to migrate actively). Experiments to validate our findings can thus be easily conducted in in-vitro studies with an experimental setting similar to that in Ref. [10] by growing tumor spheroids in a granular embedding medium.

\section{GEOMETRIC SHAPE PROPERTIES}

\section{A. Morphological Operators}

All datasets were given as binary 3D images, which are generally defined as the quadruple $P=\left(\mathbb{Z}^{3}, m, n, B\right)$, where every element of $\mathbb{Z}^{3}$ is a point (voxel) in $P$. The set $B \subseteq \mathbb{Z}^{3}$ is the image foreground, or the object, whereas $\mathbb{Z}^{3} \backslash B$ is the background. The neighborhood relation between the voxels is given by $m$ and $n$ with $m$ being the connectivity of object voxels and $n$ the connectivity of the background. To avoid topological paradoxa, only the following combinations are possible: $(6,26),(26,6),(6,18)$ and $(18,6)$ [15].

Morphological operators are well-known in image processing. Erosion and Dilation are in fact binary convolutions with a mask describing the background-connectivity of a voxel [11]. The Hit-Miss-Operator extracts specific features of a binary image. For morphological Thinning, this operator is used with a set of masks, where each mask is applied to the original image, and all resulting images undergo a logical OR-operation and will be subtracted from the original image [15].

\section{B. Distance Transform}

The distance field of a binary digital image is a discrete scalar field of the same size with the property, that each value of the scalar field specifies the shortest distance of the voxel to the boundary of the object. The signed distance transform contains negative values for distances outside the object. Distance transforms using the $L_{1}$ or $L_{\infty}$ metrics can be computed using Erosion for successive border generation and labeling of the removed voxels until the object is completely removed [11]. The computation of the Euclidean distance transform is described in [19].

\section{Medial Axis Transform and Skeletonization}

In a continuous space, the medial axis of an object is the set of points, which are the centers of maximally inscribed spheres. A sphere is maximally inscribed, if it touches the object boundary in at least two points, if it lies completely within the object, and if there is no larger sphere with the same properties. The skeleton of a binary object is a compact representation of its geometry and shape. It is a subset of the object with three properties [17]: (1) topological equivalence, (2) thinness, and (3) central location within the object. Topological equivalence implies that the medial axis has the same number of connected components, enclosed background regions and holes as the original object.

In discrete space, the medial axis can be approximated by iterative Thinning as described in [15]. 


\section{Distance Histogram and Compactness}

For quantifying the tumor surface as well as the geometry of the medial axis, the object voxels of the medial axis image were used as set of representatives for picking the corresponding scalar values from the distance field. Based on the definition of the medial axis in continuous space, the histogram displays the distribution of the object's inscribed spheres' radii as presented on the left hand side of Figures 4 and 8 .

To obtain a different set of representatives, the distance transform's ridge lines [6] were computed and rasterized into another binary image, which was again used to pick distance field values for the histogram. Since ridge lines run along local maxima and crests of a scalar field, the voxelized ridge lines are voxels locally centered within the object, and are therefore a similar representation as the medial axis in terms of geometry.

We used the distance histogram of the medial axis to compare different datasets with each other. The histogram's frequency values were interpreted as vector, and the $L_{2}$-Norm was computed between any two datasets to reveal similarities based on the medial axis. A fixed histogram interval for all datasets was applied. To avoid distortions due to different scales, the simulated tumor objects were rasterized in such a way, that the objects' bounding boxes had approximately the same dimensions.

For spheroid objects, such as the tumor growth simulation data, a third type of histogram is presented: the distribution of distances from the object's barycenter to all boundary voxels (Figure 5). The set of 26-connected boundary voxels of the image $G$ can be extracted using Erosion and set difference:

$\partial_{26} G=G \backslash\left(G \ominus M_{26}\right)$ using the $3 \times 3 \times 3$ mask $M_{26}$, where all voxels are 0 except the center voxel and its 6 -connected neighbors. For an Euclidean sphere, the histogram would show only one bar at the sphere's radius and a very small deviation due to voxelization artifacts.

A further rather simple measure applied on the tumor objects is the compactness as a normalized area/volume ratio being $C=\frac{A^{3}}{36 \pi V^{2}}$ for $3 \mathrm{D}$ objects. For all bounded objects, it is always $C \geq 1$, and $C=1$ for a Euclidean sphere. Though simple, this measure has shown to be a good criterion to evaluate the complexity of tumor surfaces. A different description of compactness is given in [5], termed discrete compactness: $C_{D}=\frac{A_{C}}{A_{C \max }}$, and was applied on histological data in [4]. $A_{C}$ denotes the number of voxel contact surfaces within the object consisting of $n \geq 2, n \in \mathbb{N}$, voxels, and $A_{C \max }=3\left(n-n^{(2 / 3)}\right)$ being the theoretical maximum of surface contacts on a given voxel number. According to this measure, a cube has the discrete compactness $C_{D}=1$.

\section{TOPOLOGICAL SHAPE PROPERTIES}

The terms of Algebraic Topology used in the following section are in detail explained in [20]. Topologically, the medial axis transform is a retraction process, where a three-dimensional simplicial complex is mapped to a twodimensional simplicial complex consisting of line segments and triangles only. The $k$-th Betti number $\beta_{k}$ of a simplicial complex $\mathscr{K}$ is the rank of its $k$-dimensional homology group:

$$
\beta_{k}:=\operatorname{dim} H_{k}(\mathscr{K})=\operatorname{dim} \frac{C_{k}(\mathscr{K})}{I_{k}(\mathscr{K})}, k=0,1,2, \cdots
$$

where $C_{k}(\mathscr{K})$ is the kernel, and $I_{k}(\mathscr{K})$ is the image of the boundary operator, that maps $k$-simplices to their $(k-1)$ chains. $C_{k}(\mathscr{K})$ is also termed the $k$-th cycle group, since its elements are unbounded $k$-dimensional simplicial complexes or $k$-cycles.

For a volume embedded in $3 \mathrm{D}, \beta_{0}$ is the number of connected components, $\beta_{1}$ is the number of independent tunnels through the volume, and $\beta_{2}$ is the number of voids enclosed by the volume. The medial axis - topologically representing the volume - is a two-dimensional simplicial complex embedded in 3D space, therefore only the Betti numbers $\beta_{k}$ with $k \leq 2$ are non-zero. Betti numbers are invariant among all simplicial complexes triangulating the same topological space.

On our binary tumor images, $\beta_{0}$ and $\beta_{2}$ can easily be determined by flood fill operations on a 26 -connected foreground $\left(\beta_{0}\right)$, and on a 6 -connected background $\left(\beta_{2}\right)$, respectively, and counting the necessary operations. As for $\beta_{1}$, further processing of the voxelized medial axis is necessary. Once the number of voids is determined, the corresponding enclosing surfaces of the medial axis have to be "punctured" by removing any one object voxel, that separates two background components under the condition, that the number of connected foreground components remains the same. Further Thinning is applied to the medial axis until all surfaces are collapsed and only line segments are left, resulting in a onedimensional simplicial complex. The voxel representation is now converted into a skeleton graph according to [18]. We performed a depth-first-search on this graph to obtain the number of independent cycles being $\beta_{1}$ and minimized this cycle base by linearly combining cycles and creating elements with shorter path length. This is necessary for characterizing individual cycles in terms of path length and position within the object.

\section{RESULTS}

\section{A. Simulation Data}

In the computer simulations, we varied the motility, the initial density, and the adhesiveness of the embedding cells. All simulated tumor samples were discretized on a $256^{3}$ voxel grid with equally sized bounding boxes. We evaluated growth simulation data of spheroid tumor models with 9 different parameter configurations. Two datasets (asym_mot 1 and asym_mot 20) developed in an anisotropic environment, resulting in asymmetric tumors. Figures 2 and 3 show the renderings of two simulated tumor objects (id120_mot1 and id100_mot30) with different growth behavior. The id-value, id120 denotes that the initial cell density is $100 / 120$ of the reference value id100, and the cell's motility mot-value, mot 30 indicates a 30 times lower motility than mot1. For Fig. 2 the cell population size was below the critical size at which a necrotic core forms while for Fig. 3 


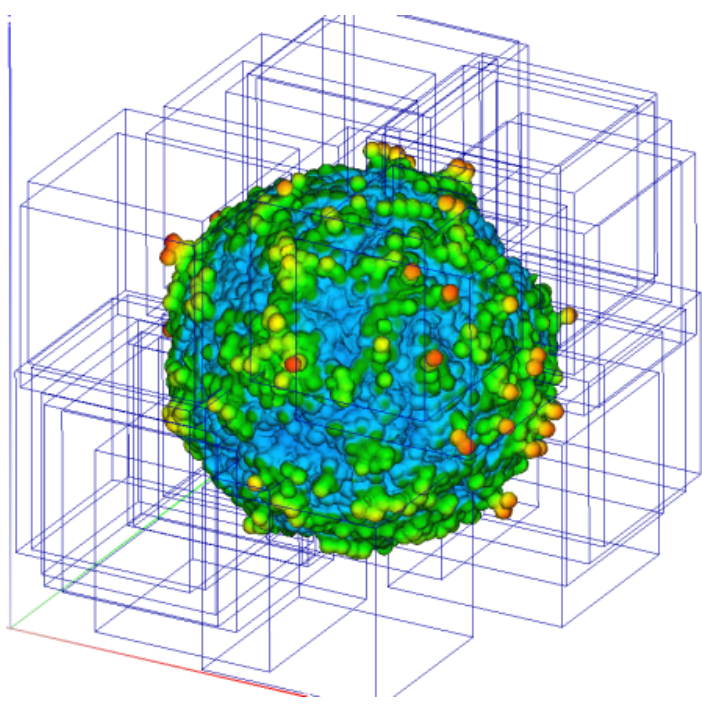

Fig. 2. Boundary of simulated tumor sample id120_mot1. The bounding boxes of 30 random samples are shown.

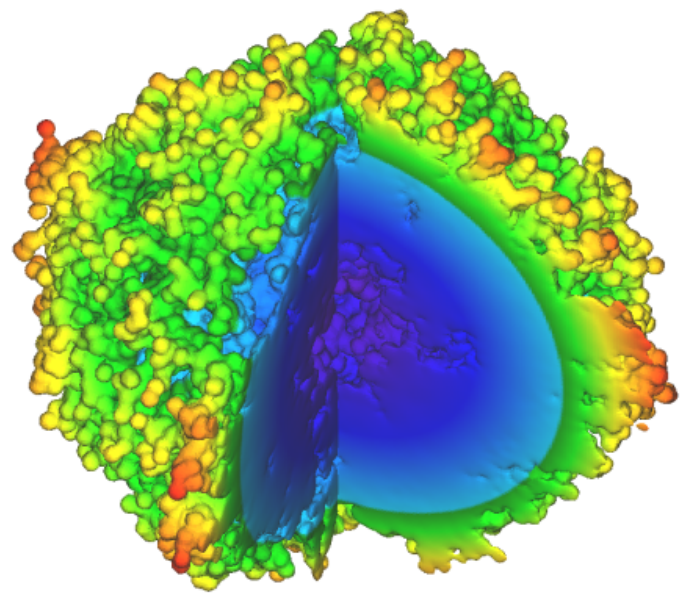

Fig. 3. Boundary of simulated tumor sample id100_mot30. The inner surface due to the necrotic core is shown.
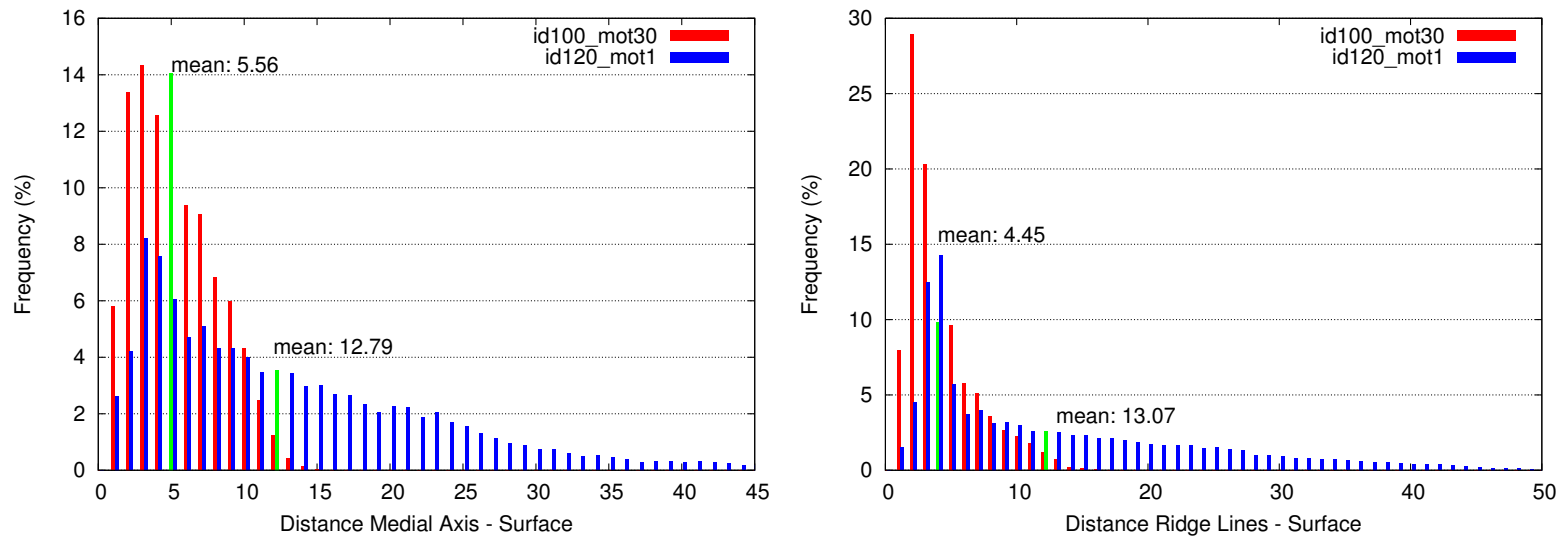

Fig. 4. Distance histograms of sample id120_mot1 and sample id100_mot30. L.h.s.: radii of the inscribed medial axis spheres; r.h.s.: ridge line points to nearest boundary. The green bars indicate the interval with the sample's average distance.
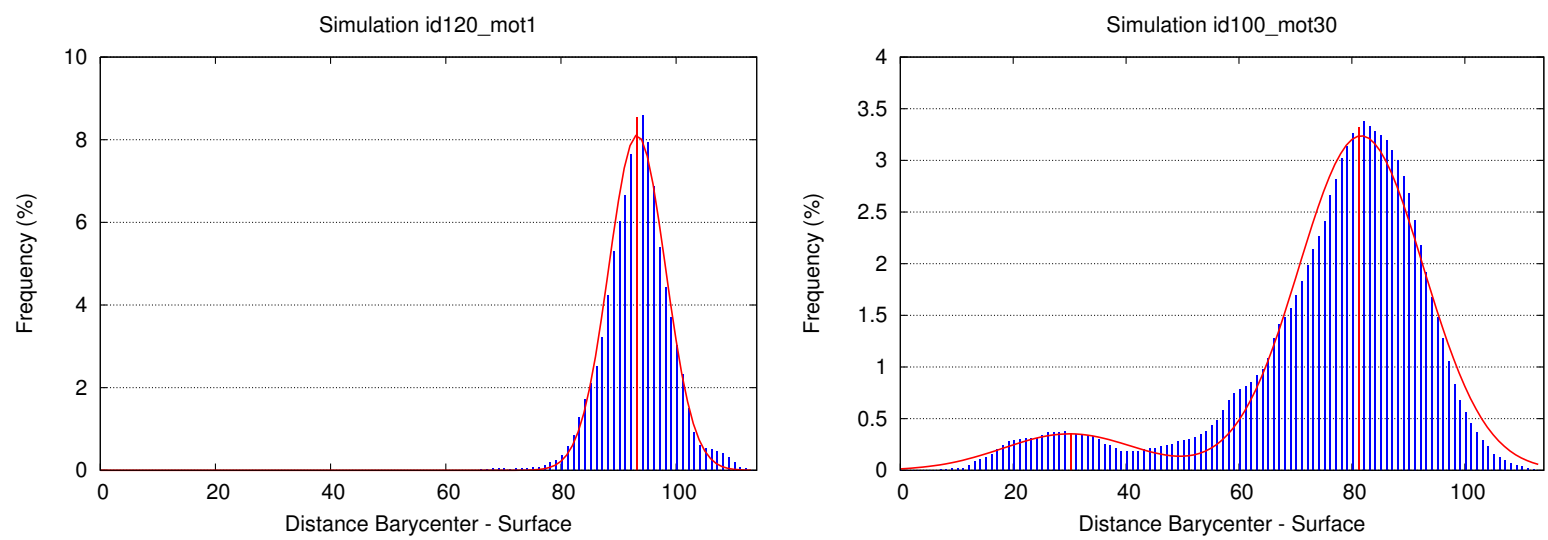

Fig. 5. Histograms of the distance values from the objects' barycenter to the surface. L.h.s.: We approximated the data with a Gaussian distribution (red) with $\mu=93.24$ and $\sigma^{2}=24.21$ using the least square fit method. R.h.s.: The tumor object has a centrally located necrotic core, thus creating a significant portion of inner surface, which shows up as second maximum. In this case, the weighted sum of two Gaussian distributions $\left(\mu_{1}=30, \sigma_{1}^{2}=138.9\right.$ and $\mu_{2}=81.659, \sigma_{2}^{2}=121.8$ ) fits the data if the first Gaussian is weighted with 0.1 and the second with 0.9 , respectively. 


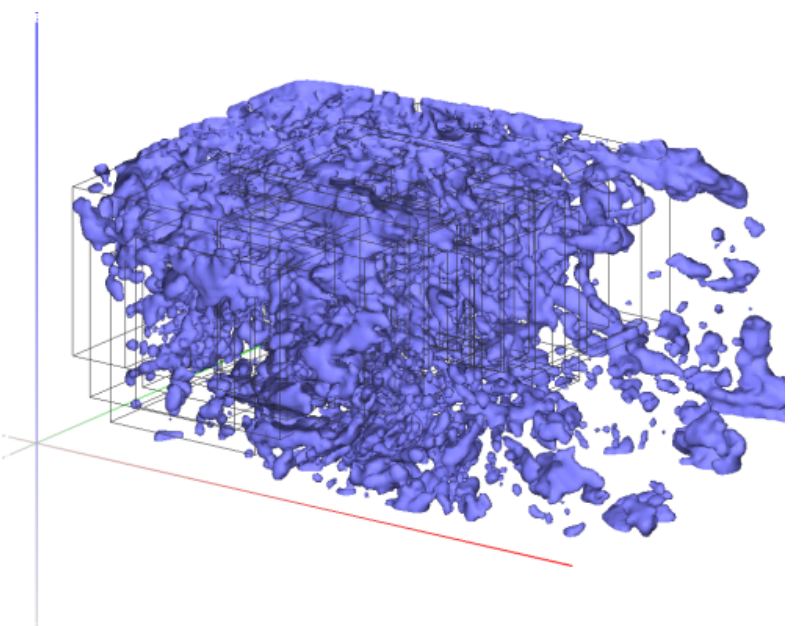

Fig. 6. Boundary of cervix carcinoma specimen 11 (diffuse tumor type). The bounding boxes of 30 random samples containing approx. $13 \%$ foreground voxels, are shown.

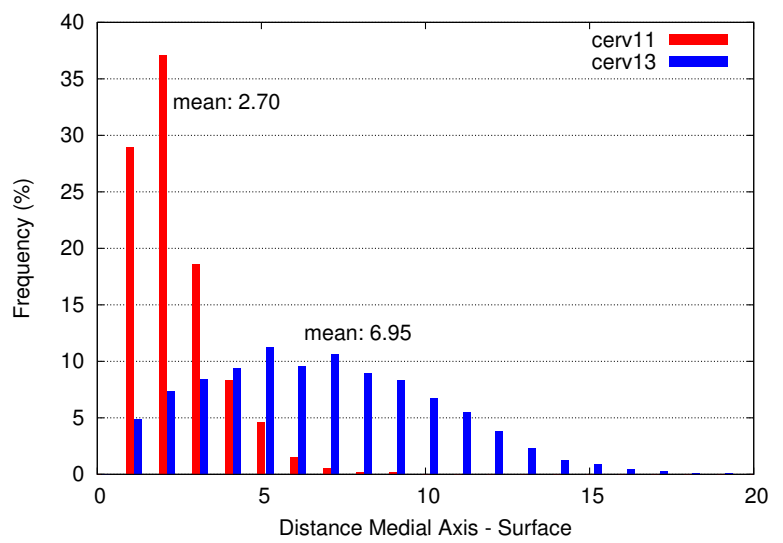

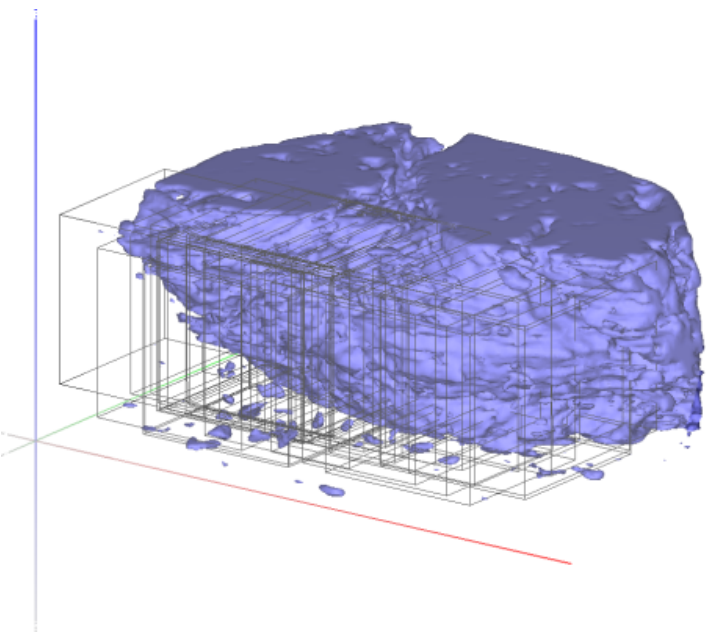

Fig. 7. Boundary of cervix carcinoma specimen 13 (compact tumor type). The bounding boxes of 30 random samples containing approx. $27 \%$ foreground voxels, are shown. The object contains many small cavities not visible in this representation.

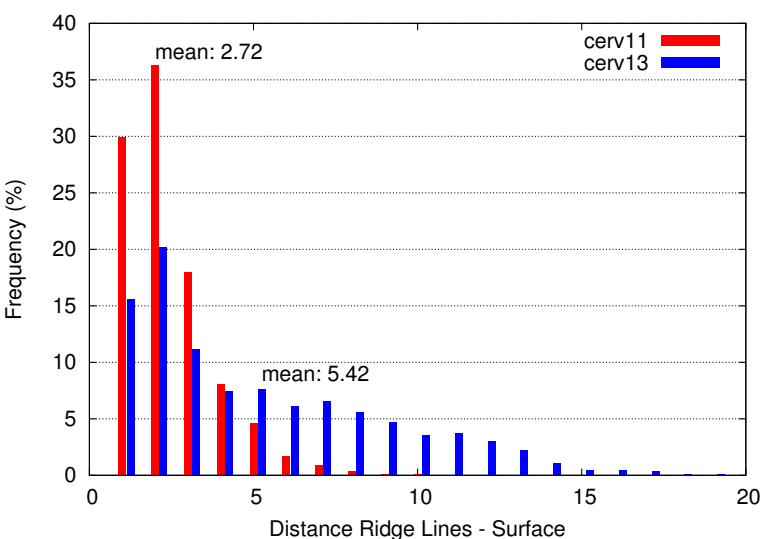

Fig. 8. Distance histograms of the two cervix carcinoma specimen. L.h.s.: radii of the inscribed medial axis spheres; r.h.s.: ridge line points to nearest boundary. For specimen 11, the object consists of many small components, each with a separate medial axis, which is - in this case - very similar to the discretized ridge lines. The mean distance values are shown.

TABLE I

RESULTS OF THE COMPACTNESS COMPUTATION AND TOPOLOGICAL ANALYSIS.

\begin{tabular}{|c|c|c|c|c|c|c|c|c|c|c|c|c|}
\hline \multirow[b]{2}{*}{ Dataset } & \multicolumn{7}{|c|}{ Average of 30 Samples } & \multicolumn{5}{|c|}{ Complete Object } \\
\hline & Area & Volume & Com & ctness & $\beta_{0}$ & $\beta_{1}$ & $\beta_{2}$ & Comp & tness & $\beta_{0}$ & $\beta_{1}$ & $\beta_{2}$ \\
\hline 1. asym_mot1 & 15618.5 & 198596 & 0.85 & 0.9876 & 1.00 & 11.01 & 0.00 & 6.67 & 0.9929 & 1 & 176 & 0 \\
\hline 2. asym_mot20 & 28815.9 & 192697 & 5.70 & 0.9717 & 1.07 & 33.03 & 0.00 & 60.41 & 0.9773 & 2 & 624 & 0 \\
\hline 3. id095_mot1 & 27406.1 & 199477 & 4.57 & 0.9750 & 1.43 & 40.08 & 0.00 & 36.86 & 0.9809 & 6 & 468 & 0 \\
\hline 4. id100_mot1 & 24598.7 & 190235 & 3.64 & 0.9766 & 1.33 & 35.39 & 0.00 & 28.55 & 0.9835 & 5 & 475 & 0 \\
\hline 5. id100_mot10 & 33698.5 & 189090 & 9.46 & 0.9662 & 1.37 & 43.27 & 0.00 & 74.92 & 0.9723 & 5 & 475 & 0 \\
\hline 6. id100_mot20 & 34658.0 & 185317 & 10.72 & 0.9647 & 1.07 & 40.03 & 0.00 & 100.39 & 0.9688 & 2 & 447 & 0 \\
\hline 7. id100_mot30 & 46132.2 & 184418 & 25.53 & 0.9507 & 1.00 & 288.88 & 1.59 & 254.65 & 0.9564 & 1 & 2867 & 17 \\
\hline 8. id100_mot1_adh & 32872.8 & 195883 & 8.19 & 0.9680 & 1.03 & 101.50 & 0.00 & 66.16 & 0.9768 & 2 & 1301 & 0 \\
\hline 9. id120_mot1 & 18581.0 & 200296 & 1.41 & 0.9844 & 1.00 & 24.27 & 0.00 & 10.76 & 0.9903 & 1 & 316 & 0 \\
\hline 10. cerv11 (diffuse type) & 89644.6 & 134022 & 354.66 & 0.8800 & 110.43 & 101.11 & 0.75 & 3337.46 & 0.8742 & 934 & 618 & 5 \\
\hline 11. cerv13 (compact type) & 55138.9 & 273947 & 19.75 & 0.9601 & 50.37 & 106.11 & 36.41 & 114.21 & 0.9758 & 334 & 1284 & 755 \\
\hline
\end{tabular}

a necrotic core has formed. The corresponding histograms in Figure 4 show the distance distribution from the medial axis (1.h.s.) and ridge lines (r.h.s.) to the surface, and the distances from the object's barycenter to the surface points, respectively (Figure 5). Besides a difference in the distribution of the distances between medial axis and object surface, the absolute distance values are significantly larger for the tumor in Fig. 2 than for the tumor in Fig. 3. This can be explained by more invasive finger-shaped patterns in the latter case. The less complex the medial axis, the 
TABLE II

Distance Matrix of the Medial Axis Histogram's Pairwise $L_{2}$-Norms.

\begin{tabular}{|c|c|c|c|c|c|c|c|c|c|c|c|}
\hline & 1 & 2 & 3 & 4 & 5 & 6 & 7 & 8 & 9 & 10 & 11 \\
\hline 1 & - & 0.1664 & 0.0459 & 0.0361 & 0.2122 & 0.2181 & 0.2810 & 0.1166 & 0.0351 & 0.5696 & 0.2067 \\
\hline 2 & & - & 0.1582 & 0.1482 & 0.1149 & 0.1088 & 0.2138 & 0.1241 & 0.1618 & 0.5804 & 0.0976 \\
\hline 3 & & & - & 0.0269 & 0.1883 & 0.1975 & 0.2547 & 0.0927 & 0.0477 & 0.5534 & 0.1998 \\
\hline 4 & & & & - & 0.1883 & 0.1960 & 0.2579 & 0.0875 & 0.0320 & 0.5545 & 0.1931 \\
\hline 5 & & & & & - & 0.0287 & 0.1030 & 0.1203 & 0.1974 & 0.5150 & 0.0873 \\
\hline 6 & & & & & & - & 0.1129 & 0.1326 & 0.2044 & 0.5255 & 0.0694 \\
\hline 7 & & & & & & & - & 0.1800 & 0.2604 & 0.4450 & 0.1601 \\
\hline 8 & & & & & & & & - & 0.0927 & 0.4997 & 0.1460 \\
\hline 9 & & & & & & & & & - & 0.5431 & 0.1967 \\
\hline 10 & & & & & & & & & & - & 0.5516 \\
\hline 11 & & & & & & & & & & & - \\
\hline
\end{tabular}

smoother the corresponding histogram. The frequency of small distance values is always higher due to the fanning-out of the medial axis towards the object boundary resulting from voxelization artifacts. The histogram depicting the distances from the object's barycenter to the boundary voxels (Fig. 5) suggests a connection between a complex surface and a higher variance of the value distribution, which could be confirmed by fitting a Gaussian Distribution to the data in the case id120_mot1 and a weighted sum of two Gaussians in the case id100_mot30. The variance $\sigma^{2}=24.21$ in the first case is approximately one fifth of the two variance values $\sigma_{1}^{2}=138.9, \sigma_{2}^{2}=121.8$ in the latter. On the right hand side of Figure 5, a second maximum at small values indicates the necrotic core's surface close to the center of the object.

Table II shows the pairwise Euclidean distances between the medial axis histograms. The lower left half of the symmetric matrix visualizes the similarity values as gray boxes with black indicating the smallest, and white indicating the largest distance. The three most similar dataset pairs are highlighted in bold.

Table I presents the results of the compactness computation and topological analysis. Four simulated datasets have been compared regarding different motility properties $(\operatorname{mot}=1,10,20,30)$, and three datasets regarding different cell densities (initial cell distance: $i d=95,100,120$ ). One tumor object (id100_mot1_adh) developed in an environment with modified embedding cell adhesion properties, resulting in a more complex morphology as suggested by the topological results $\left(\beta_{1}\right)$ and the compactness values shown in Table I. The first seven columns depict average values over 30 random samples containing approximately the same amount of foreground voxels $(20 \pm 5 \%)$. The last five columns show the results of the complete object. There are two compactness measures shown, the first being the normalized continuous surface/volume ratio with a minimum of 1 for a maximally compact object (sphere), whereas the second is the described discrete compactness with a maximum of 1 for the cube. Hence, a large first and small second value both indicate an increasing invasive structure. Note, that there is a compactness value of 0.85 in the first row, which would be impossible according to the above definition. The sample generation introduced "fake" surfaces, which were not taken into account. This is also the reason, why the first compactness value is generally smaller for the samples than for the complete objects. The compactness measures indicate a decreasing compactness with decreasing motility, increasing initial density of the embedding cells, and if cell-cell adhesion between the embedding cells is present. This is physically plausible. A decreasing motility inhibits a uniform spread of the tumor which would require a uniform outward displacement of the embedding cells. Instead, the expanding tumor sprouts into regions where the local density of embedding cells is smaller and form invasive fingers. With increasing density, the embedding cells are more difficult to displace and it is more favorable for the expanding cell clone to sprout into the spaces resulting from inhomogeneities of the spatial distribution of the embedding cells. If embedding cells are adherent they form locally dense aggregates which are difficult to push aside such that the expanding cell clone again sprouts into the space between those aggregates. Note that the Betti number $\beta_{2}$ shows the same tendencies as the first compactness measure, while the Betti number $\beta_{1}$ is insensitive to the interface pattern between tumor and surrounding medium. The Betti number $\beta_{2}$ proves useful to identify cavities. It is non-zero only for id100_mot 30 , where a hollow core emerged as a consequence of necrosis. Hence, $\beta_{2}$ and the distance between barycenter and surface are both suited to identify large cavities.

In summary, the geometric and topological measures permit an excellent quantification of the invasiveness of the simulated tumors and are in perfect agreement with the biophysical intuition.

\section{B. Histological tumor material}

For comparison of our results from the simulation data, we additionally evaluated two datasets obtained from a 3D reconstruction of a series of histological slice images from cervix carcinoma [4] (Figures 6 and 7). The specimen represent two different types of in-vivo tumor growth: diffuse and compact. The diffuse type consisted of approximately 1.2 million cells, was about $4.1 \mathrm{~mm} \times 2.9 \mathrm{~mm} \times 1.5 \mathrm{~mm}$ 
in size, and was discretized on a 414 x $291 \times 153$ voxel grid. The compact type had about 4.6 million cells with a size of $3.1 \mathrm{~mm} \times 2.9 \mathrm{~mm} \times 1.3 \mathrm{~mm}$ and was presented on a grid of $311 \times 291 \times 127$ voxels. Although the morphology of the compact type seems to be much simpler than the one of the diffuse type, the Betti numbers $\beta_{1}$ and $\beta_{2}$ suggest a high complexity, invisible in the $3 \mathrm{D}$ rendering of the object (Figure 7).

The histological datasets do not represent the complete tumor. Instead, they show only a portion, and artificial surfaces were introduced. To avoid biased results caused by those surfaces, 30 cuboid sections containing the surface or region of interest were extracted, evaluated, and the averaged results were compared with the average of 30 random cuboid samples extracted from the simulation data. To determine the samples' positions, for each voxel position the foreground/background ratio was computed given the chosen sample size (100 x $100 \times 100$ voxels). Among the positions with ratio values falling into the desired interval ( $13 \pm 5 \%$ for the diffuse type, $27 \pm 5 \%$ for the compact type), 30 positions were randomly chosen to extract the samples.

As for the diffuse tumor type (cerv11), according to the histogram shown in Figure 8, the medial axis is very similar to the discretized ridge lines. This can be explained by the object's morphology, suggested by $\beta_{0}$. The object consists of many small components, each with a separate medial axis with, in this case, the same properties as ridge lines.

\section{CONCLUSIONS AND FUTURE WORKS}

We have adapted and tested different techniques to evaluate and quantify the morphology of tumor objects. We compared growth simulations with different parameter configurations with each other, and adapted the described methods for evaluating in-vivo tumor objects as well. The obvious differences in complexity observed in the 3D renderings of the object surfaces could be confirmed and quantified with the methods described. Though sensitive to noise at the object's boundary, the medial axis has proven to be suitable for representing the dataset's shape and geometry. In summary, the used geometric and topological measures proved useful to quantify rough boundaries and infiltration pattern of tumors indicating invasive behavior. In a next step, we will apply different methods for shape description, such as principal component and momentum analysis. As additional histological and simulated datasets are available, the presented methods will be statistically analyzed in more detail to establish classification methods of tumor growth behavior. Comparing the distance histograms by means of interpreting them as vectors and computing the pairwise $L_{2}$ Norm, similarities between different tumor shapes could be identified. The presented distance matrix suggests a method for clustering the datasets based on the similarity of their medial axes. Moreover the time development of the measures during the growth of a tumor will be studied.

\section{ACKNOWLEDGMENTS}

The authors are thankful to J.-P. Kuska and U.-D. Braumann for providing the histological data reconstructed from in-vivo tissue samples. We also thank the FAnToM development team, as many of the visualization methods are based on the FAnToM framework. S.H. acknowledges support by the BMBF-grant Hepato Sys project 0313081, D.D. from the DFG-grant BIZ 6-1/1.

\section{REFERENCES}

[1] N. Amenta, S. Choi, and R. Kolluri. The power crust, unions of balls, and the medial axis transform. Computational Geometry: Theory and Applications, 19(2-3):127-153, 2001.

[2] A. Bonnassie, F. Peyrin, and D. Attali. Shape description of threedimensional images based on medial axis. Image Processing, 3:931934, 2001.

[3] G. Borgefors, I. Nystrom, and G. S. di Baja. Computing skeletons in three dimensions. Pattern Recognition, 32(7):1225-1236, 1999.

[4] U.-D. Braumann, J.-P. Kuska, J. Einenkel, L.-C. Horn, M. Löffler, and M. Höckel. Three-Dimensional Reconstruction and Quantification of Cervical Carcinoma Invasion Fronts From Histological Serial Sections. IEEE Transactions on Medical Imaging, 24(10):1286-1307, 2005.

[5] E. Bribiesca. A measure of compactness for 3-D shapes. Comput. Math. Applicat., 40:1275-1284, 2000.

[6] Y.-C. Chang and A. Frigeri. Implementing the automatic extraction of ridge and valley axes using the PPA algorithm in Grass GIS. In Proceedings of the Open source GIS, 2002.

[7] N. D. Cornea, D. Silver, and P. Min. Curve-skeleton applications. Visualization, 2005, pages 95-102, 2005.

[8] D. Drasdo and S. Höhme. A single-cell-based model of tumor growth in vitro: monolayers and spheroids. Physical Biology, 2:133-147, 2005.

[9] Y.-S. C. et al. Johnson-Kendall-Roberts Theory Applied to Living Cells. Phys. Rev. Lett., 280:312, 1999.

[10] G. Helmlinger, P. Netti, H. Lichtenfeld, R. Melder, and R. Jain. Solid stress inhibits the growth of multicellular tumor spheroids. Nature Biotechnology, 15(8):778-783, 1997.

[11] B. Jähne. Digital Image Processing. Springer Verlag, Berlin, 2005.

[12] K. Johnson, K. Kendall, and A. Roberts. Surface energy and the contact of elastic solids. Proc. R. Soc. London A, 324:301, 1971.

[13] K. J. Kruszynski, R. van Liere, and J. A. Kaandorp. An interactive visualization system for quantifying coral structures. EUROVIS Eurographics /IEEE VGTC Symposium on Visualization, pages 283290, 2006.

[14] L. Kunz-Schughart. Multicellular tumor spheroids: intermediates between monolayer culture and in vivo tumor. Cell Biology International, 23(3):157 - 161, 1999.

[15] K. Palágyi and A. Kuba. A 3d 6-subiteration thinning algorithm for extracting medial lines. Pattern Recognition Letters, 19(7):613-627, 1998.

[16] V. Pascucci and K. Cole-McLaughlin. Efficient computation of the topology of level sets. VIS '02: Proceedings of the conference on Visualization '02, pages 187-194, 2002.

[17] C. Pudney. Distance-ordered homotopic thinning: A skeletonization algorithm for $3 \mathrm{~d}$ digital images. Computer Vision and Image Understanding, 72(3):404-413, 1998.

[18] F. Reinders, M. E. D. Jacobson, and F. H. Post.

[19] T. Saito and J. Toriwaki. Algorithms of three dimensional Euclidean distance transformation and extended digital Voronoi diagram, and analysis of human liver section images. The Journal of the Institute of Image Electronics Engineers of Japan, 21(5):468-474, 1992.

[20] R. Stöcker and H. Zieschang. Algebraische Topologie. Teubner Verlag, Stuttgart, 1994.

[21] R. M. Sutherland. Cell and environment interactions in tumor microregions:the multicell spheroid model. Science, 240:177-184, 1988.

[22] R. Weinberg. The biology of cancer. Garland Science, New York and Oxford, 2007. 